\title{
I ncidentes críticos no processo de ensino-aprendizagem em diabetes na perspectiva da equipe multiprofissional de saúde
}

\section{Critical incidents in the teaching-learning process in diabetes in the perspective of a multiprofessional health team}

\section{Incidentes críticos en el proceso de enseñanza-aprendizaje en diabetes en la perspectiva del equipo multiprofesional de salud}

\begin{abstract}
Camila Rezende Pimentel Ribas', Carla Regina de Souza Teixeira", Valmir Aparecido de Oliveira"', Tatiane Aparecida Martins ${ }^{\prime \mathrm{V}}$, Karina Dal Sasso Mendes ${ }^{\mathrm{v}}$, Nájela Hassan Saloum de Andrade ${ }^{\mathrm{VI}}$, Manoel Antônio dos Santos ${ }^{\mathrm{VII}}$, Maria Lúcia Zanetti ${ }^{\mathrm{VII}}$
\end{abstract}

\section{RESUMO}

Estudo descritivo transversal que teve como objetivo identificar os fatores que interferem positiva e negativamente no processo de ensino-aprendizagem de diabéticos, na perspectiva da equipe multiprofissional de saúde. Foi realizado em um Centro de pesquisa e extensão universitária do interior paulista em 2007. Participaram 11 sujeitos, membros da equipe multiprofissional do referido centro. Para coleta de dados utilizou-se a técnica de incidentes críticos, com aplicação de um formulário. Os resultados indicaram 15 incidentes críticos, sendo oito com referências positivas e 11 negativas. Os fatores mais valorizados por tais sujeitos integrantes dessa equipe de saúde na educação em diabetes foram: interação entre os pacientes no grupo, interação da equipe multiprofissional de saúde e acolhimento da equipe. Dentre os fatores menos valorizados constatou-se a interação da equipe multiprofissional de saúde, além da configuração do grupo e dificuldade do profissional em lidar com situações adversas no grupo. Os dados obtidos remetem à necessidade de investimento das instituições formadoras em saúde para capacitar profissionais com competência para trabalhar com grupos, discutindo os fatores que favorecem positiva e negativamente na educação em diabetes.

Palavras chave: Diabetes Mellitus; Educação em saúde; Equipe interdisciplinar de saúde.

\section{ABSTRACT}

This descriptive and cross-sectional study aimed to identify factors that interfere either positively or negatively in diabetes patients' teachinglearning process, from the perspective of the multidisciplinary health team. It was carried out at a university research and community service center in the interior of São Paulo State, Brazil in 2007. Eleven subjects participated, who are members of the center's multidisciplinary team. The critical incident technique was used for data collection, through the application of a form. The results indicated 15 critical incidents, eight with positive and 11 with negative references. The factors the health professionals most valued in diabetes education were: interaction among patients in the group, interaction in the multiprofessional health team and welcoming by the team. The least valued factors included interaction in the multidisciplinary health team, group configuration and professionals' difficulty

Psicóloga, mestranda em Enfermagem Fundamental pela Escola de Enfermagem de Ribeirão Preto - EERP da Universidade de São Paulo - USP, bolsista Capes. Ribeirão Preto, SP. E-mail: camilar.p.ribas@usp.br.

"Professor Doutor do Departamento de Enfermagem Geral e Especializada da EERP-USP, Ribeirão Preto, SP. E-mail: carlarst@eerp.usp.br.

III Psicólogo, mestrando em Enfermagem Fundamental pela EERP-USP. Membro da equipe de Educação em Diabetes vinculado à EERP-USP. E-mail: valoliveir@hotmail.com.

IV Graduanda em Licenciatura em Enfermagem pela EERPUSP. Membro da equipe de Educação em Diabetes vinculado à EERP-USP. E-mail: tatiane.martins@usp.br.

${ }^{\vee}$ Enfermeira, doutoranda em Enfermagem Fundamental pela EERP-USP. Membro da equipe de Educação em Diabetes vinculado à EERP-USP. E-mail: dalsasso@eerp.usp.br.

VI Enfermeira, doutoranda em Enfermagem Fundamental pela EERP-USP. Membro da equipe de Educação em Diabetes vinculado à EERP-USP. E-mail: najela@ibest.com.br.

VII Professor Doutor do Departamento de Psicologia e Educação da Faculdade de Filosofia, Ciências e Letras de Ribeirão Preto (FFCLRP) da Universidade de São Paulo. Membro da equipe de Educação em Diabetes vinculado à EERP-USP. E-mail: masantos@ffclrp.usp.br.

VIII Professor Associado do Departamento de Enfermagem Geral e Especializada da EERP-USP. Presidente da Comissão de Pesquisa da EERP-USP, coordenadora do Grupo de Pesquisa em Enfermagem e Diabetes Mellitus cadastrado no diretório do CNPq. Coordenadora do Grupo de Educação em Diabetes vinculado à EERP-USP. E-mail: zanetti@eerp.usp.br. 
to deal with adverse situations in the group. The collected data reveal the need for investments by health education institutions, in order to train professionals for working with groups, discussing the factors that exert positive and negative effects on diabetes education.

Key words: Diabetes Mellitus; Health education; Patient care team.

\section{RESUMEN}

Estudio descriptivo transversal con objeto de identificar los factores que interfieren positiva y negativamente en el proceso de enseñanzaaprendizaje de diabéticos, en la perspectiva del equipo multiprofesional de salud. Fue realizado en un centro de investigación y extensión universitaria del interior del estado de São Paulo, Brasil en 2007. Participaron 11 sujetos, miembros del equipo multiprofesional del referido centro. Para la recolecta de datos se utilizó la técnica de incidentes críticos, con

\section{NTRODUÇÃO}

O diabetes é um problema de saúde pública de grande importância, não só a nível nacional, mas também mundial, em virtude do aumento de sua prevalência e incidência. As repercussões sociais e econômicas comprometem a produtividade, a qualidade de vida e a sobrevida dos indivíduos, contribuindo, assim, para o aumento das aposentadorias precoces, dos custos do tratamento e das suas complicações $^{(1-2)}$. Na década de 1990, o diabetes afetou a saúde de 110 milhões de indivíduos e até 2010 estima-se que esse número poderá dobrar para 221 milhões $^{(3)}$.

As projeções no Brasil para 2025 apontam para a existência de cerca de 11 milhões de diabéticos, o que representa um aumento de $100 \%$ em relação aos atuais 5 milhões de indivíduos $^{(3)}$. Há vários fatores que contribuem para o aumento da morbi-mortalidade dessa doença. $\mathrm{Na}$ maioria dos países, aproximadamente $50 \%$ dos pacientes desconhecem sua doença e por isso não se tratam, e os 20 a $30 \%$ que a conhecem apresentam pouca adesão ao tratamento ${ }^{(4)}$.

O tratamento da pessoa com diabetes tem como finalidade obter um bom controle metabólico, considerando que a falência do aplicación de un formulario. Los resultados indicaron 15 incidentes críticos, siendo ocho con referencias positivas y 11 negativas. Los factores más valorizados por los profesionales de salud en la educación en diabetes fueron: interacción entre los pacientes en el grupo, interacción del equipo multiprofesional de salud y acogimiento del equipo. Los factores menos valorizados incluyeron la interacción del equipo multiprofesional de salud, configuración del grupo y dificultad del profesional en lidiar con situaciones adversas en el grupo. Los datos obtenidos exponen la necesidad de inversión de las instituciones formadoras en salud para capacitar profesionales con competencia para trabajar con grupos, discutiendo los factores que favorecen positiva y negativamente la educación en diabetes.

Palabras clave: Diabetes Mellitus; Educación en salud; Grupo de atención al paciente.

tratamento tem como conseqüências, a curto e longo prazo, complicações agudas e crônicas.

Dois estudos importantes realizados com pacientes diabéticos, tipo $1^{(5)}$ e tipo $2^{(6)}$, demonstraram uma redução nas complicações quando os pacientes receberam controle intensivo de insulina, sob orientação de uma equipe multiprofissional capacitada para esse fim, o que reforça a importância da educação em diabetes como caminho para o alcance do bom controle metabólico.

Assim, a educação em diabetes como ferramenta para a obtenção do bom controle metabólico baseia-se na freqüência apropriada de automonitorização da glicose sangüínea, na terapia nutricional, na atividade física regular, na terapêutica farmacológica, nas instruções sobre a prevenção e o tratamento das complicações crônicas e agudas, na educação e avaliação contínuas dos objetivos do tratamento, bem como o acompanhamento psicológico.

No entanto, apesar do considerável progresso tecnológico e científico alcançado nos últimos anos na área de diabetes, a educação do paciente tem sido o recurso mais enfatizado no cuidado ao paciente frente à complexa abordagem terapêutica instituída. Por outro 
lado, a avaliação de educação ao paciente ainda é um desafio para os profissionais de saúde ${ }^{(7)}$.

A avaliação contínua e abrangente deve ser obrigatória para que seja determinado se os objetivos estabelecidos foram alcançados nos programas de educação em diabetes. 0 resultado do programa educativo para pacientes com diabetes tem sido avaliado em termos de prevalência de complicações ou de variáveis clínicas. Estudos têm demonstrado o efeito benéfico da educação no controle glicêmico, como a redução do número de hospitalizações, de amputações dos membros inferiores, das taxas de complicações agudas e do aparecimento e progressão de complicações crônicas da doença ${ }^{(5-8)}$.

A avaliação da educação do paciente diabético deve estar articulada no processo de avaliação da qualidade geral do cuidado em diabetes. A educação é uma ferramenta que ajuda positivamente os pacientes durante seu tratamento, mas sua eficácia varia de acordo com o aspecto da doença a ser controlado e as mudanças comportamentais necessárias. Muito esforço tem sido empregado no desenvolvimento de instrumentos válidos e apropriados para avaliação dos programas de educação em diabetes ${ }^{(8)}$.

Um exame crítico e abrangente da educação em saúde, durante as últimas décadas, aponta para o desenvolvimento e a reorientação crescente das reflexões teóricas e metodológicas nesse campo de estudo. Pode-se destacar a contribuição das Ciências Sociais e dos estudos de Antropologia da Saúde contemporâneos $^{(9)}$.

A ação educativa fundamenta-se na adoção de um modo de vida natural e adequado, a fim de controlar a doença ${ }^{(9)}$. Essa estratégia educacional tem um grande impacto sobre o comportamento das pessoas com diabetes e até mesmo nos custos de atendimento à saúde em diabetes. Estudos mostraram que as mudanças no estilo de vida por meio da educação dos diabéticos resultam em redução de peso, melhor controle glicêmico, da pressão arterial e lipídios e, conseqüentemente, reduzem os riscos cardiovasculares $^{(5-6)}$. Assim, é importante que as pessoas diabéticas adquiram o conhecimento sobre as ferramentas de autocuidado em diabetes para tomar as decisões diárias no seu cotidiano.

Considerando o caráter assintomático da doença, motivar as pessoas diabéticas, com níveis glicêmicos alterados, quando elas não apresentam ainda nenhum sinal ou sintoma da doença, é um dos desafios que o profissional de saúde tem que enfrentar no cuidado com essa clientela. Para isso, as estratégias educacionais devem atender aos aspectos emocionais, sociais, de valores e crenças dessas pessoas em relação à própria saúde ${ }^{(10)}$.

Portanto, é necessário que a equipe multiprofissional seja capacitada e qualificada para 0 atendimento considerando essas premissas, o que tem sido recomendado por vários pesquisadores ${ }^{(11-12)}$. Um dos aspectos centrais para a capacitação da equipe multiprofissional é o conhecimento das tecnologias leve e leve-dura para o atendimento de grande demanda da clientela diabética.

Dentre as tecnologias que os profissionais necessitam dominar incluem-se os fatores que podem interferir no processo ensinoaprendizagem para aquisição de habilidades cognitivas, motoras e afetivas.

Diante do exposto esse estudo se propõe identificar os fatores que interferem positiva e negativamente no processo de ensinoaprendizagem de diabéticos, na perspectiva da equipe multiprofissional de saúde.

\section{METODOLOGI A}

Estudo descritivo transversal realizado em um Centro de Pesquisa e Extensão Universitária do interior paulista, em 2007. A população do estudo foi constituída por oito profissionais e três alunos de graduação, integrantes do Grupo de Educação em Diabetes (GED) do referido Centro.

Nesse estudo foi adotada como procedimento de coleta de dados a técnica do incidente crítico. Essa técnica consiste em um conjunto de procedimentos para coleta de observações diretas do comportamento humano, facilitando sua utilização potencial na solução de problemas práticos e no desenvolvimento de amplos princípios psicológicos. Essa técnica delineia ainda, 
procedimentos para a coleta de incidentes observados que apresentem significação especial e para o encontro de critérios sistematicamente definidos ${ }^{(13)}$. Incidente referese a "qualquer atividade humana observável que seja completa em si mesma para permitir inferências e previsões a respeito da pessoa que executa o ato. Para ser crítico, um incidente deve ocorrer em uma situação onde o propósito ou intenção do ato pareça razoavelmente claro ao observador, onde suas conseqüências sejam suficientemente definidas para deixar poucas dúvidas no que se refere aos seus efeitos"(13).

A essência da técnica do incidente crítico implica em solicitar ao observador ou aos sujeitos envolvidos numa determinada atividade, tipos simples de julgamentos ou relatos de situações e fatos, que serão avaliados pelos pesquisadores em função da concordância ou não que possuem com o objetivo e a natureza da atividade ou situação que se almeje estudar.

Para coleta de dados elaborou-se um formulário contendo duas partes: a primeira, com dados de identificação dos sujeitos (sexo, idade, formação profissional, tempo de formação) e a segunda, com duas questões norteadoras para identificação dos aspectos que interferem positiva e negativamente no processo de ensino-aprendizagem dos diabéticos durante um programa de educação em diabetes.

O instrumento foi avaliado por dois especialistas em diabetes e submetido a um pré-teste com três profissionais. Após a adequação do instrumento, a coleta de dados foi realizada no período de maio a junho de 2007, no próprio Centro, mediante preenchimento do formulário pelo próprio participante, após a assinatura do Termo de Consentimento Livre e Esclarecido.

Para obtenção dos dados referentes aos incidentes críticos foi solicitado que os participantes descrevessem situações ocorridas durantes as atividades desenvolvidas no Programa Educativo em Diabetes, que interferiam positiva e negativamente no processo de ensino-aprendizagem.

Para organização dos dados obtidos com a técnica de incidentes críticos faz-se necessária uma sistematização das informações, evitandose que essas observações sejam realizadas ao acaso, sem metodologia, e freqüentemente dependendo somente da inferência do observador ${ }^{(13)}$. Nessa direção, a análise dos dados obtidos obedeceu aos quatro critérios propostos, a saber: leitura e arrolamento dos relatos; identificação dos elementos que compreendem 0 incidente crítico (situação, comportamento e conseqüência); agrupamentos dos relatos e categorização de situação, comportamentos e conseqüências ${ }^{(13)}$. No presente estudo, os dados foram analisados separadamente como relatos de incidentes, com referências positivas ou negativas.

O projeto foi aprovado pelo Comitê de Ética em Pesquisa da Escola de Enfermagem de Ribeirão Preto, protocolo no 0667/2006.

\section{RESULTADOS}

Dos 11 sujeitos, a maioria era do sexo feminino $(72,7 \%)$, sendo que $54,5 \%$ encontravam-se entre 18 e 29 anos (Tabela 1). 
Ribas CRP, Teixeira CRS, Oliveira VA, Martins TA, Mendes KDS, Andrade NHS et al. Incidentes críticos no processo de ensinoaprendizagem em diabetes na perspectiva da equipe multiprofissional de saúde. Rev. Eletr. Enf. [Internet]. 2008; 10(3):74755. Available from: http://www.fen.ufg. br/revista/v10/n3/v10n3a20.htm.

Tabela 1: Distribuição numérica e percentual da equipe multiprofissional de saúde, segundo o sexo, formação profissional, tempo de formação e idade. Ribeirão Preto-SP, 2007.

\begin{tabular}{|c|c|c|}
\hline Sexo & $\mathbf{n}$ & $\%$ \\
\hline $\begin{array}{l}\text { Feminino } \\
\text { Masculino }\end{array}$ & $\begin{array}{l}8 \\
3\end{array}$ & $\begin{array}{l}72,7 \\
27,3\end{array}$ \\
\hline I dade & $\bar{n}$ & $\%$ \\
\hline $\begin{array}{l}18 \text { a } 29 \text { anos } \\
30 \text { a } 39 \text { anos } \\
40 \text { a } 49 \text { anos } \\
50 \text { a } 59 \text { anos }\end{array}$ & $\begin{array}{l}6 \\
4 \\
- \\
1\end{array}$ & $\begin{array}{c}54,5 \\
36,4 \\
- \\
9,1\end{array}$ \\
\hline Formação Profissional & $\bar{n}$ & $\%$ \\
\hline $\begin{array}{c}\text { Enfermagem } \\
\text { Estudantes de psicologia } \\
\text { Educador físico } \\
\text { Nutrição }\end{array}$ & $\begin{array}{l}4 \\
3 \\
2 \\
2\end{array}$ & $\begin{array}{l}36,4 \\
27,3 \\
18,2 \\
18,2\end{array}$ \\
\hline Tempo de formação & $\mathbf{n}$ & $\%$ \\
\hline $\begin{array}{c}0 \text { a } 5 \text { anos } \\
6 \text { a } 10 \text { anos } \\
\text { Mais de } 10 \text { anos } \\
\text { Em formação }\end{array}$ & $\begin{array}{l}4 \\
3 \\
1 \\
3\end{array}$ & $\begin{array}{c}36,4 \\
27,3 \\
9,1 \\
27,3\end{array}$ \\
\hline Total & 11 & 100 \\
\hline
\end{tabular}

A Tabela 2 mostra os incidentes críticos encontrados nos relatos dos sujeitos. Foram encontradas 15 ocorrências, sendo oito com referências positivas e 11 negativas. Os relatos mais encontrados foram: nove referentes aos incidentes críticos positivos e quatro negativos, relacionados à interação dos pacientes no grupo e interação da equipe multiprofissional de saúde, respectivamente.

Tabela 2: Distribuição numérica dos incidentes críticos positivos e negativos na perspectiva da equipe multiprofissional de saúde relacionados ao processo de ensino-aprendizagem. Ribeirão Preto-SP, 2007.

\begin{tabular}{ccc} 
Incidentes críticos & Positivos & Negativos \\
\hline Interação entre os pacientes no grupo & 9 & 2 \\
Interação da equipe multiprofissional de saúde & 6 & 4 \\
Acolhimento da equipe & 3 & - \\
Perfil metabólico dos pacientes & 2 & - \\
Configuração do grupo & 2 & - \\
Mensuração de parâmetros clínicos & 1 & - \\
Interesse dos pacientes no programa & 1 & 2 \\
Capacitação do profissional em diabetes & 1 & 1 \\
Falta de conhecimento das complicações crônicas & - & 1 \\
no processo de mudança de comportamento dos pacientes & - & 1 \\
de do profissional em lidar com situações adversas no grupo & - & 1 \\
Descontinuidade do atendimento & - & 2 \\
Mau controle metabólico dos pacientes & - & 1 \\
Recursos materiais & - & 1
\end{tabular}

\section{SCUSSÃO}

As diretrizes da Sociedade Brasileira de Diabetes recomendam que a educação em diabetes deve integrar atendimento clínico, promoção de saúde, aconselhamento, manejo da doença e pesquisa(11). Para o manejo do diabetes a equipe multiprofissional de saúde deve estar constituída no mínimo por um enfermeiro e um nutricionista, podendo também dela participar educadores físicos, psicólogos, farmacêuticos e médicos, com capacitação em diabetes $^{(11)}$. Quanto à caracterização dos sujeitos do estudo, encontrou-se que a composição do grupo de educação do referido Centro está em consonância com as diretrizes da Sociedade Brasileira de Diabetes, o que pode ser considerado uma composição diferenciada para o atendimento do paciente diabético.

A inclusão de alunos de graduação - no caso, de Psicologia - mostra a preocupação da 
Ribas CRP, Teixeira CRS, Oliveira VA, Martins TA, Mendes KDS, Andrade NHS et al. Incidentes críticos no processo de ensinoaprendizagem em diabetes na perspectiva da equipe multiprofissional de saúde. Rev. Eletr. Enf. [Internet]. 2008; 10(3):74755. Available from: http://www. fen.ufg. br/revista/v10/n3/v10n3a20.htm.

equipe com a inserção precoce do estudante na prática clínica, o que possibilita formar profissionais críticos, uma das metas propostas pelas instituições formadoras na área da saúde. A vivência nos grupos educativos em diabetes pode contribuir para o amadurecimento do estudante no processo de ensino-aprendizagem, viabilizando ferramentas para o enfrentamento das dificuldades e os desafios que os futuros profissionais enfrentarão no exercício profissional.

Na perspectiva da equipe multiprofissional do referido Centro, o incidente crítico positivo mais evocado foi a interação dos pacientes no grupo. Sabe-se que tanto a interação quanto o apoio mútuo entre os integrantes do grupo contribuem para o aumento da motivação para a busca do cuidado em saúde, o que irá favorecer mudanças de atitudes frente à doença e, conseqüentemente, melhora no controle metabólico e no bem-estar geral ${ }^{(14)}$. Assim, o apoio psicológico, o suporte e os esclarecimentos oferecidos por todos os profissionais da equipe são identificados como essenciais para fortalecer os pacientes a desenvolverem estratégias de enfrentamento desde o estabelecimento do diagnóstico do paciente ${ }^{(14)}$. Por outro lado, os relatos de incidente crítico negativo referente à interação da equipe multiprofissional de saúde mostram que o trabalho em equipe também assume uma conotação negativa para alguns participantes.

A interação entre os profissionais da equipe de saúde também foi referida pelos sujeitos como positiva, do ponto de vista da viabilização do processo de ensinoaprendizagem. Esses relatos destacaram a importância das reuniões semanais da equipe multiprofissional, por contribuírem para maior sintonia dos profissionais envolvidos. Os profissionais de saúde são vistos como responsáveis por facultar condições favorecedoras ao processo de aquisição de conhecimentos científicos acerca do controle do diabetes $^{(14) \text {. }}$

A convivência da equipe multiprofissional de saúde pode permitir que os pacientes usufruam de uma visão mais ampla dos seus problemas, fortalecendo sua motivação e conhecimentos para superar os desafios impostos pela doença e tratamento, favorecendo a adoção de atitudes para mudanças de hábitos de vida, com melhor adesão ao tratamento proposto(15).

Uma vez que a interação dos pacientes no grupo é um fator que, na perspectiva dos profissionais de saúde, favorece o alcance do êxito nos programas de educação em diabetes, deve ser estimulada e reforçada dentre as estratégias que motivam para o autocuidado, utilizadas na elaboração dos programas ${ }^{(12)}$.

O perfil metabólico do paciente também foi referido pelos sujeitos como positivo. Esse caráter positivo foi relatado à medida que os diabéticos adquiriam maior controle, tendem a dar continuidade ao programa educativo, sentindo-se mais dispostos e motivados para a aprendizagem.

Como para atingir a meta do bom controle metabólico é necessário manter a freqüência apropriada de monitorização da glicemia capilar, a terapia nutricional, a atividade física regular, os esquemas terapêuticos farmacológicos, as informações acerca da prevenção e tratamento das complicações crônicas e agudas, e o reforço e avaliação periódica dos objetivos do tratamento ${ }^{(16)}$, atingir o perfil metabólico é um dado que favorece a educação em diabetes, na perspectiva dos profissionais do referido Centro.

A mensuração dos parâmetros clínicos foi relatada como um incidente positivo que favorece a educação em diabetes. Assim, recomenda-se a mensuração da glicemia capilar, do peso corporal, da altura e da circunferência abdominal em todos os atendimentos em diabetes, pois a dinâmica desses procedimentos pode constituir-se em um elemento motivador para a mudança de hábitos e modificação de estilo de vida, tão fundamentais para o controle da doença ${ }^{(17-18)}$.

Por outro lado, a descontinuidade do atendimento apareceu como um incidente crítico que interfere negativamente no processo de ensino-aprendizagem. Essa descontinuidade foi associada ao recesso de férias dos profissionais, às precárias condições materiais e de equipamentos utilizados no atendimento à pessoa diabética e o comparecimento irregular às atividades propostas no Centro. 
Ribas CRP, Teixeira CRS, Oliveira VA, Martins TA, Mendes KDS, Andrade NHS et al. Incidentes críticos no processo de ensinoaprendizagem em diabetes na perspectiva da equipe multiprofissional de saúde. Rev. Eletr. Enf. [Internet]. 2008; 10(3):74755. Available from: http://www. fen.ufg. br/revista/v10/n3/v10n3a20.htm.

Assim, a adequação de recursos materiais quanto ao número, integridade e calibramento dos equipamentos, o local apropriado quanto a conforto, iluminação e ruído, a organização de escala de férias da equipe, os motivos que levam ao absenteísmo dos pacientes ao grupo são componentes que devem estar sempre presentes na pauta de discussão da equipe quando da proposição de programas de educação(19).

O acolhimento por parte da equipe multiprofissional aos diabéticos integrantes dos grupos foi mencionado como positivo, na medida em que propicia condições favoráveis ao processo ensino-aprendizagem devido ao estímulo à expressão de seus problemas, medos, anseios, perspectivas futuras, desejos e fantasias.

Para um acolhimento eficaz e satisfatório, é preciso capacitar a equipe de saúde para atender as necessidades específicas inerentes ao controle metabólico do paciente, com a responsabilidade que esse trabalho envolve. Nesse sentido, entende-se que o acolhimento transcende a simples postura compreensiva e empática do profissional, implicando em ações efetivas que contribuam para retirar o paciente do risco no caso de uma glicemia alterada, ou encaminhamento adequado em cada situação específica. Quando os profissionais desenvolvem - acolhimento baseado nessas premissas há possibilidade de fortalecer a confiança e o vínculo do paciente com os profissionais. Por outro lado, os profissionais têm disponibilidades diferentes ao iniciarem um trabalho grupal. Esse fato pode interferir no aproveitamento das oportunidades de aprendizagem e pode trazer ao usuário maior ou menos satisfação com a interação ${ }^{(20)}$.

O interesse dos diabéticos no programa educativo foi relatado como positivo. A motivação intrínseca dos usuários, na perspectiva dos profissionais de saúde, é um fator facilitador para a compreensão e adesão ao tratamento instituído. Para pacientes com diabetes, a motivação em relação ao programa educativo é normalmente acompanhada por suas observações acerca de complicações ou variáveis clínicas ${ }^{(8)}$. 0 interesse espontâneo do paciente tem de ser explorado pelos profissionais como um fator que favorece a aprendizagem; por outro lado, é necessário desenvolver estratégias ativas para sensibilizar e envolver aqueles que ainda não despertaram para a importância do controle metabólico.

As dificuldades da equipe em lidar com as situações adversas no grupo de diabéticos e a qualidade da interação entre os pacientes no grupo foram mencionadas como fatores que interferem negativamente no processo de ensino-aprendizagem. As dificuldades da equipe em enfrentar adversidades que emergem no grupo de pacientes são situações que muitas vezes extrapolam os conhecimentos técnicos específicos de cada área profissional e exigem aportes de outra natureza, como habilidades relacionais. A qualidade das relações interpessoais estabelecidas pelos pacientes com os demais membros do grupo pode constituir também em barreiras para a educação em diabetes, pois as trocas de experiências e conhecimentos ficam restritas e não possibilitam a reflexão, reelaboração e incorporação de conceitos e habilidades requeridas no cotidiano dos usuários.

A configuração do grupo de diabéticos foi referida tanto positiva quanto negativamente. $\mathrm{Na}$ perspectiva dos profissionais de saúde a homogeneidade do grupo em relação ao diagnóstico de diabetes contribuiu favoravelmente com o processo de ensinoaprendizagem face à troca de experiências, dificuldades similares, favorecendo o vínculo e, conseqüentemente, o apoio mútuo entre os membros do grupo.

Também foi enfatizado que a formação de grupos com 10 a 12 membros por grupo propicia maior participação, interação, exposição e esclarecimento de dúvidas e, conseqüentemente, maior aproveitamento. Possibilita também à equipe um olhar mais individualizado e maior disponibilidade de atenção ao diabético do que um grupo com número extenso de pacientes. I mporta ressaltar que um grupo numeroso pode tornar inviável a comunicação e contribuir para formação de grupos paralelos ou subgrupos.

A capacitação do profissional em diabetes foi apontada como fator positivo na educação na perspectiva da equipe de saúde. As 
habilidades, a formação e a qualificação dos profissionais foram percebidas como relevantes no processo de ensino-aprendizagem.

\section{CONSI DERAÇÕES FI NAIS}

A utilização da técnica de incidente crítico possibilitou identificar que os fatores que, na perspectiva dos profissionais da equipe de saúde, contribuem positivamente para a educação em diabetes foram: interação entre os pacientes no grupo, interação da equipe multiprofissional de saúde, acolhimento da equipe, perfil metabólico dos pacientes, configuração do grupo, mensuração dos parâmetros clínicos, interesse dos pacientes no programa e capacitação do profissional em diabetes.

Os fatores que contribuem negativamente para o processo de ensino-aprendizagem dos diabéticos foram: interação da equipe multiprofissional de saúde, interação entre os pacientes no grupo, capacitação do profissional em diabetes, configuração do grupo, mau controle metabólico dos pacientes, falta de conhecimento das complicações crônicas, demora no processo de mudança de comportamento dos pacientes, dificuldade do profissional em lidar com situações adversas no grupo, descontinuidade do atendimento e recursos materiais.

No que concerne aos fatores mais valorizados pelos profissionais de saúde na educação em diabetes, constatou-se a interação entre os pacientes no grupo, interação da equipe multiprofissional de saúde e acolhimento da equipe; dentre os fatores menos valorizados, situam-se a interação da equipe multiprofissional de saúde, configuração do grupo e dificuldade do profissional em lidar com situações adversas no grupo.

A interação entre os pacientes e a interação dos membros da equipe de saúde foi evidenciada com muita ênfase, valorizando os aspectos biológicos, sociais, psicológicos, ambientais, econômicos, espirituais, entre outros, da capacitação profissional, fundamentais à manutenção da atenção integral à saúde.

Considerando a alta prevalência do diabetes, bem como a agenda da saúde na atualidade no Brasil, que preconiza que a atenção aos usuários deve ser oferecida por equipe multiprofissional, os dados obtidos remetem à reflexão de que há necessidade de investimento das instituições formadoras em saúde para capacitar profissionais com competência para trabalhar com grupos, discutindo os fatores que favorecem positiva e negativamente na educação em diabetes.

\section{REFERÊNCI AS}

1. Sociedade Brasileira de Diabetes. Consenso Brasileiro de diagnóstico e classificação do diabetes mellitus e tratamento do diabetes mellitus tipo 2. São Paulo (Brasil): Sociedade Brasileira de Diabetes; 2001.

2. American Association Of Diabetes Educators. White paper on the prevention of type 2 diabetes and the role of the diabetes educator. The Diabetes Educator. 2002; 28(6):964-71.

\section{International Diabetes Federation.} Complicações do diabetes e educação. Diabetes Clínica. 2002;6(3):217-20.

4. Malerbi DA, Franco LJ. Multicenter study of the prevalence of diabetes mellitus and impaired glucose tolerance in the urban Brazilian population aged 30-69 years. Diabetes Care. 1992;15(11): 1509-16.

5. The Diabetes Control and Complications Trial Research Group. The Effect of Intensive Treatment of Diabetes on the Development and Progression of Long-Term Complications in Insulin-Dependent Diabetes Mellitus. The New England Journal of Medicine. 1993; 329(14): 977-86.

6. United Kingdom Prospective Diabetes Study (UKPDS) Group. Intensive blood-glucose control with sulfonylureas or insulin compared with conventional treatment and risk of complications in patients with type 2 diabetes. The Lancet. 1998;352(9131):837-53.

7. Mühlhauser I, Berger M. Patient education evaluation of a complex intervention. Diabetologia. 2002; 45(12): 1723-33.

8. Tankova T, Dakovska G, Koev D. Education and quality of life in diabetic patients. Patient Education and Counseling. 2004;53(3):285-90.

9. Gazzinelli MF, Gazzinelli A, Reis DC, Penna CMM. Educação em saúde: conhecimentos, representações sociais e experiências da 
doença. Cadernos de Saúde Pública. 2005; 21(1):200-06.

10. Teixeira CRS, Zanetti ML. O trabalho multiprofissional com grupo de diabéticos. Revista Brasileira de Enfermagem. 2006;59(6): 812-7.

11. Sociedade Brasileira de Diabetes. Tratamento e acompanhamento do diabetes mellitus: diretrizes da Sociedade Brasileira de Diabetes. Rio de Janeiro (Brasil): Sociedade Brasileira de Diabetes; 2007.

12. Ferraz AEP, Zanetti $M L$, Brandão ECM, Romeu LC, Foss MC, Paccola GMGF, et al. Atendimento multiprofissional ao paciente com diabetes mellitus no Ambulatório de diabetes do HCFMRP-USP. Medicina. 2000; 33(2):170-5.

13. Flanagan JC. A técnica do incidente crítico. Arquivos brasileiros de psicologia aplicada. 1973;25(2): 99- 141

14. Zanetti ML, Biaggi MV, Santos MA, Peres DS, Teixeira CRS. O cuidado à pessoa diabética e as repercussões na família. Revista Brasileira de Enfermagem. 2008: (61): 186-92.

15. Sociedade Brasileira de Cardiologia, Sociedade Brasileira de Hipertensão, Sociedade Brasileira de Nefrologia (orgs.). V Diretrizes brasileiras de hipertensão arterial. São Paulo (Brasil): Sociedade Brasileira de Cardiologia; 2006.

16. American Diabetes Association. Nutrition recommendations and interventions for diabetes-2006: a position statement of the American Diabetes Association. Diabetes Care. 2006; 29(9): 2140-57.

17. Swinburn BA, Metcalf PA, Ley SJ. Long-term (5-year) effects of a reduced-fat diet intervention in individuals with glucose intolerance. Diabetes Care 2001;24(4):619-23.

18. Tuomilehto J, Lindström J, Eriksson JG, Valle $T$, Hämäläinen $H$, llanne-Parikka $P$, et al. Prevention of type 2 diabetes mellitus by changes in lifestyle among subjects with impaired glucose tolerance. The New England J ournal of Medicine. 2001;344(18): 1343-50.

19. Teixeira CRS, Zanetti ML, Martins DC. Parâmetros clínicos de pacientes diabéticos atendidos no Programa educativo do Serviço de Medicina Preventiva-SEMPRE. Ciência, Cuidado e Saúde. 2005: (4):231-41.
20. Gagliardino JJ, Etchegoyen G. A model educational program for people with type 2 diabetes: a cooperative Latin American implementation study (PEDNID-LA). Diabetes Care 2001; 24(6): 1001-7.

Artigo recebido em 28.02.08

Aprovado para publicação em 30.09.08 